\title{
Outcome in Triple Valve Surgery: Our Experience
}

\author{
N. Gautam, B. Rajbanshi, S. Pradhan, J. Sharma, R. Koirala, B. Koirala \\ Shahid Gangalal National Heart Center
}

\section{Objective}

Shahid Gangalal National Heart Centre does about 1200 cardiac operations per year. Valve Surgery especially for rheumatic valves comprises about half of the operations performed every year. Over 1500 valve surgeries have already been performed in the centre since 2002 till date. We present our experience on triple valve surgery.

\section{Method}

All the patients who underwent triple valve surgery between August 2001 and July 2007 were studied. There were 58 such patients. Mean age was $30.29 \pm 12.5$, (range:13 to 68 years). There were 37 (60\%) male patients. Immediate postoperative complications, mortality and follow up data were studied. All the patients were evaluated in three months and one year postoperatively by chest X-ray, ECG and Electrocardiography. Follow Up data was available for all the 51 surviving patients at 3 months and for 42 patients at 1 year.

\section{Result}

Two (3.8\%) patients had to undergo re-exploration for excessive bleeding. Four (7.1\%) patients died in early postoperative period. Significant number of patients had improved NYHA functional class at one year of follow up. There was no prosthetic valve endocarditis, prosthetic valve thrombosis and paravalvular leak.

\section{Conclusion}

Triple valve surgery can be performed with satisfactory early and intermediate Postoperative results. 